\title{
Reiterated Targeting Peptides on the Nanoparticle Surface Significantly Promote Targeted Vascular Endothelial Growth Factor Gene Delivery to Stem Cells
}

\author{
Dong-Dong Wang ${ }^{\dagger, \ddagger}$, Mingying Yang ${ }^{\S,},{ }^{,}$, , Ye Zhu ${ }^{\dagger}$, and Chuanbin Mao ${ }^{\dagger,}$, \\ tDepartment of Chemistry and Biochemistry, Stephenson Life Sciences Research Center, \\ University of Oklahoma, Norman, Oklahoma 73019, United States \\ \$Institute of Applied Bioresource Research, College of Animal Science, Zhejiang University, \\ Hangzhou, Zhejiang 310058, China
}

\begin{abstract}
Nonviral gene delivery vectors hold great promise for gene therapy due to the safety concerns with viral vectors. However, the application of nonviral vectors is hindered by their low transfection efficiency. Herein, in order to tackle this challenge, we developed a nonviral vector integrating lipids, sleeping beauty transposon system and 8-mer stem cell targeting peptides for safe and efficient gene delivery to hard-to-transfect mesenchymal stem cells (MSCs). The 8-mer MSCtargeting peptides, when synthetically reiterated in three folds and chemically presented on the surface, significantly promoted the resultant lipid-based nanoparticles (LBNs) to deliver VEGF gene into MSCs with a high transfection efficiency $(\sim 52 \%)$ and long-lasting gene expression (for longer than $170 \mathrm{~h}$ ) when compared to nonreiterated peptides. However, the reiterated stem cell targeting peptides do not enable the highly efficient gene transfer to other control cells. This work suggests that the surface presentation of the reiterated stem cell-targeting peptides on the nonviral vectors is a promising method for improving the efficiency of cell-specific nonviral gene transfection in stem cells.
\end{abstract}

\section{Graphical abstract}

*Corresponding Authors. yangm@zju.edu.cn.cbmao@ou.edu.

\#D.-D.W. and M.Y. contributed equally to this work.

ASSOCIATED CONTENT

Supporting Information

The Supporting Information is available free of charge on the ACS Publications website at DOI: 10.1021/acs.biomac.5b01226.

Schematic illustration of transposon system. DLS and zeta potential data of liposomes containing VT-peptide. DNA retardation assay to determine the DNA loading in protamine at various weight ratios of protamine/DNA. Gel retardation assay of LBNs at various liposome/DNA mass ratios. MTT assay of rMSCs at different concentrations of LBNs and Lipofectamine 2000. Transfection efficiencies of LBNs with and without 3VT-peptide (PDF).

The authors declare no competing financial interest. 


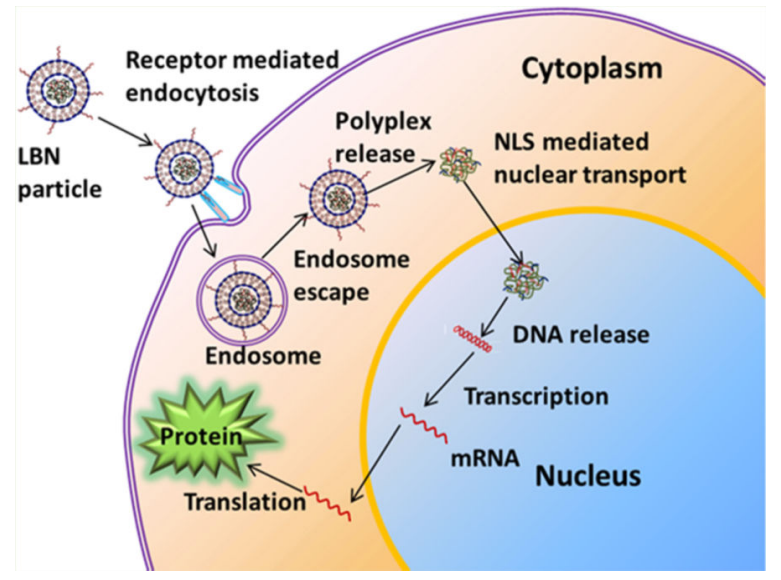

\section{INTRODUCTION}

Stem cell-based gene therapy is a promising approach to the treatment of many diseases such as myocardial ischemia, ${ }^{1,2}$ bone defects, ${ }^{3,4}$ and cancer. ${ }^{5,6}$ In this approach, a foreign gene, such as the one encoding vascular endothelial growth factor (VEGF), is carried by stem cells such as mesenchymal stem cells (MSCs) and the transfected stem cells are implanted into the diseased sites (e.g., ischemic heart or bone defects), followed by the expression of the gene into a functional protein (such as the VEGF that can induce blood vessel formation to repair the ischemic heart or promote bone defect healing). MSCs are considered a good cell carrier in stem cell-based gene therapy because they also have the potential to differentiate into bone, muscle, cartilage and other connective tissues. This multipluripotency makes MSCs an attractive candidate for gene therapy. ${ }^{7}$ VEGF is essential for vasculogenesis and angiogenesis to regenerate new blood vessels. ${ }^{8}$ Hence, it is a good therapeutic protein in treating diseases when new blood vessel formation is one of the keys to the success in the treatment, such as in healing damaged bones. ${ }^{9,10}$ Therefore, MSCs expressing VEGF have been used in treating various disease. ${ }^{11-13}$ As a special type of drug delivery system, ${ }^{14-17}$ gene delivery carrier is of great importance to the success in gene therapy. Viruses have been used as a carrier to deliver VEGF genes with a high efficiency. ${ }^{18-20}$ However, they are not ideal, because they can potentially induce mutagenesis and immune responses. To overcome such challenges, great efforts have been made to develop nonviral carriers for delivering genes to target cells. ${ }^{21,22}$ Nonviral vectors such as nanoparticles, ${ }^{23,24}$ cationic lipids, ${ }^{25,26}$ and polymers ${ }^{27-29}$ are proposed for VEGF gene delivery to MSCs. However, due to the lack of efficient internalization, nuclear translocation, and integration of foreign genes into host genome, the nonviral vectors generally have low transfection efficiency (typically lower than 20\%), particularly when they are used to deliver the gene into the hard-to-transfect stem cells. Thus, there is a pressing need in the development of a biocompatible and efficient nonviral vector for VEGF gene delivery to MSCs.

Previously we showed that a peptide (VTAMEPGQ, termed VT-peptide) that can target bone marrow-derived rat MSCs (rMSCs) could be selected from a random peptide library by using phage display technique. ${ }^{30}$ We also showed that when the peptide was mechanically 
mixed with lipids to form liposomes, only leaving a few molecules on the surface, the enhanced green fluorescence protein (EGFP) gene transfection efficiency could be improved from $\sim 8 \%$ to $\sim 12 \%$. We then hypothesized that if the peptide was presented on the surface of nanoparticles by chemical conjugation with the surface molecules, the nanoparticles will have a higher efficiency of recognizing the rMSCs and becoming internalized, leading to improved efficiency of delivering gene into rMSCs. Moreover, once gene is delivered into the rMSCs, the gene needs to be translocated to the cell nuclei and also inserted into the host genome for gene expression. To assist these two important steps, we adopt two measures. One is to integrate a reported nuclear localization signaling (NLS) peptide (DKKKRKV) with the DNA to be delivered. ${ }^{31,32}$ Another is to use a nontraditional special type of plasmid, a sleeping beauty (SB) transposon system, which is a mixture made of a transposon and a transposase. ${ }^{33,34}$ The transposon carries the EGFP-VEGF target gene and the transposase acts as an enzyme that can "cut" the EGFP-VEGF gene from the transposon and "paste" it into the host genome of rMSCs. The "cut-and-paste" mechanism ensures the target gene to be expressed in a long-lasting manner instead of the undesired transient manner. In essence, to solve the two daunting challenges facing the use of nonviral nanoparticles to transfect MSCs, namely, the low efficiency of delivering gene to the cells and transient production of functional protein, we allow the rMSC-targeting peptide to be chemically conjugated to the surface of lipid-based nanoparticles (LBNs), which deliver a EGFP-VEGF-encoding SB transposon system complexed with a cationic NLS peptide to rMSCs (Figure 1).

Specifically, inspired from the fact that 3 -fold repeating enhanced the peptide targeting function, ${ }^{35}$ we first employed a 3 -fold reiterated rMSC-targeting peptide

(VTAMEPGQVTAMEPGQVTAMEPGQC, termed 3VT-peptide) to study if we can observe enhanced transfection effect. Nonreiterated VTAMEPGQC (VT-peptide) was used as a control. A cysteine was introduced to the C-terminal of the peptide to facilitate peptide-lipid conjugation. Second, in contrast to the conventional plasmid vectors, the SB system provides efficient and long-term gene expression. Third, in order to overcome the nuclear membrane barrier, we incorporated the NLS peptide to enhance nuclear translocation of DNA. The NLS peptide has been proven to promote gene expression in mammalian cells. ${ }^{32,35}$ Fourth, the arginine-rich polycationic protamine is used as a condensation reagent. Studies showed that protamine was able to condense plasmid DNA efficiently to promote gene delivery to several cell types in combination with liposome. ${ }^{36,37}$ The fabricated nanoparticles displayed rMSC-targeting motif on the surface and carried an electrostatically condensed mixture of NLS peptide, protamine, and SB system inside, promoting MSC-internalization, nuclear translocation, and integration of target gene into MSCs genome, all of which collectively resulted in enhanced sustained gene expression.

\section{MATERIALS AND METHODS}

\subsection{Materials}

All the lipids, including 1,2-dioleoyl-3-trimethylammonium-propane (DOTAP), 1,2dioleoyl-sn-glycero-3-phosphoethanolamine (DOPE), and 1,2-distearoyl-sn-glycero-3phosphoethanolamine- $N$-[maleimide(polyethylene glycol)-2000] (DSPE-PEG2000-MAL), a mini extruder, and polycarbonate membrane filters were purchased from Avanti Polar Lipids 
Inc. Cholesterol, protamine, and chloroform were purchased from Sigma-Aldrich. Fetal bovine serum (FBS), Dulbecco's Modified Eagle Medium (DMEM), SYBR Safe, and lipofectamine 2000 were purchased from Invitrogen.

\subsection{Construction of VEGF SB Transposon System}

We obtained the pEGFP-VEGF plasmid containing VEGF and EGFP gene from Professor H. K. Lin (the Health Science Center, University of Oklahoma) as a gift. The original sleeping beauty transposon system, including pT2/SVNeo (the transposon) and pSB11 (the transposase), was a kind gift from Dr. Perry B. Hackett (University of Minnesota). The neomycin resistance gene in pT2/SVNeo was replaced by VEGF and EGFP gene (Scheme S1). First, DNA fragments containing VEGF and EGFP gene were amplified by PCR from pEGFP-VEGF plasmid (Primer1: GCCTGATCAATGAACTTTCTGCTGTCT; Primer2: GCCTTCGAATTACTTGTACAGCTCGTC. The underlines denote the introduced restriction enzyme sites). Second, both the DNA fragments and pT2/SVNeo plasmids were digested by restriction enzyme $B c I$ and $B s t B I$. Finally, the digested products were purified and ligated to give rise to the recombinant pT2/VEGF-EGFP plasmid. The purified pT2/ VEGF-EGFP plasmids were verified by DNA sequencing.

\subsection{Preparation of Liposomes}

The liposomes consisting of DOTAP, DOPE, and cholesterol (1:1:1 molar ratio) were prepared by thin film hydration. Briefly, a thin lipid film was formed by evaporating the lipid solvent with nitrogen gas in a $10 \mathrm{~mL}$ round-bottom flask. Hydration of the lipid films was done with vigorously vortexing for $1 \mathrm{~h}$, followed by sonication for $5 \mathrm{~min}$. The resultant liposomes were repeatedly extruded $(9 x)$ at room temperature through polycarbonate membrane filters of decreasing pore size of 0.2 and $0.1 \mathrm{~mm}$, respectively, using a mini extruder.

\subsection{Conjugation of Targeting Peptide with DSPE-PEG2000-MAL}

VTAMEPGQGGGC peptide (VT-peptide, MW $=1106.25$ ) or VTAMEPGQVTAMEPGQVTAMEPGQGC (3VT-peptide, MW = 2620.01) were synthesized by United BioSystems Inc. The reduction of cysteine residues in the targeting peptide was first performed. Briefly, $5 \mathrm{~mL}$ peptide (100 $\mu \mathrm{M}$, PBS, pH7.5) was first mixed with tris(2-carboxyethyl) phosphine (TCEP; $2 \mathrm{mM}$ ) with a 1:2 ratio of peptide/TCEP. The reaction was allowed to proceed at $37^{\circ} \mathrm{C}$ for $2 \mathrm{~h}$. A $10 \mu \mathrm{L}$ DSPE-PEG2000-MAL $(10 \mathrm{mM})$ stock solution was reacted with $5 \mathrm{~mL}(100 \mu \mathrm{M})$ of the reduced peptide in PBS buffer $(\mathrm{pH}$ 7.5), corresponding to 5:1 molar ratio of reduced peptide to maleimide groups. The conjugation reaction was carried out for $3 \mathrm{~h}$ at room temperature. Conjugated peptide-PEGDSPE was purified by dialysis (500 Da membrane).

\subsection{Preparation of Targeting LBNs}

To obtain targeting liposomes, the insertion of the peptide conjugated lipid (peptide-PEGDSPE) into liposomes was performed by employing a post-insertion technique. Targeting liposomes were prepared with varied molar percentages of peptide-PEG-DSPE $(0.1 \%, 0.5 \%$, $1 \%, 5 \%$, and $10 \%)$. For this purpose, liposome ( $20 \mathrm{mM}$, as denoted by DOTAP in liposome) 
in water solution was incubated with a various amount of peptide-PEG-DSPE for $1 \mathrm{~h}$ with vortexing. The resultant solution was kept at $60{ }^{\circ} \mathrm{C}$ for $1 \mathrm{~h}$. As a result, the conjugates became attached to the outer lipid layer of the vesicles via hydrophobic DSPE domain. The size and zeta-potential values of liposome in water were determined by dynamic light scattering (DLS; Zeta Potential Analyzer, Brookhaven Instruments Corporation, NY).

\subsection{Gel Retardation of Protamine/pDNA Polyplexes}

The protamine and pDNA $(0.25 \mu \mathrm{g})$ were mixed at different weight ratios and incubated for $20 \mathrm{~min}$. The resultant complexes were confirmed using gel electrophoresis, as shown in Figure S2 (1\% agarose gel, $105 \mathrm{~V}, 40 \mathrm{~min}$, with SYBR Safe staining).

\subsection{Gel Retardation of Liposome Protamine/DNA Lipoplexes}

The protamine and pDNA $(0.25 \mu \mathrm{g})$ were mixed at a weight ratio of 2:1 and the complex was incubated for $20 \mathrm{~min}$. Then, the DOTAP/DOPE/Chol (mole ratio 1:1:1) liposomes were added into the complex, followed by incubation for $20 \mathrm{~min}$. The resultant complexes were confirmed using gel electrophoresis, as shown in Figure S3 (1\% agarose gel, 105 V, 30 min, with SYBR Safe staining).

\subsection{Preparation of LBNs}

SB plasmids (transposon and transposase plasmids at 2:1 mass ratio), protamine, and NLS peptides were mixed at various weight ratios to form polyplex. The resultant polyplex was allowed to stand at room temperature for $30 \mathrm{~min}$. Then liposome was added into the polyplex solution followed by vortexing. The resultant mixture was incubated for $1 \mathrm{~h}$ to give rise to LBNs.

\subsection{MTT Assay}

rMSCs were isolated from bone marrow of the rats following a previously reported procedure. ${ }^{38}$ The cells were seeded at a concentration of $2 \times 10^{4}$ cells/well in 96-well plates in standard expansion medium for $24 \mathrm{~h}$. PBS was used to wash cells and different concentrations of LBNs or Lipofectamine 2000 were used to treat cells for $6 \mathrm{~h}$. Cell viability was evaluated using the 3-(4,5-dimethyl thiazol-2-yl)-2,5-diphenyl tetrazolium bromide (MTT) assay following our reported protocol. ${ }^{39} \mathrm{rMSCs}$ were incubated with MTT solution ( $5 \mathrm{mg} / \mathrm{mL}, 20 \mu \mathrm{L}$, Sigma, U.S.A.) for $4 \mathrm{~h}$. After the supernatant was discarded, dimethyl sulfoxide (DMSO, $300 \mu \mathrm{L}$ ) was used to dissolve the formazin crystals. A microplate reader (Biotek) was used to quantify cell viability by measuring absorbance at $570 \mathrm{~nm}$.

\subsection{LBN Internalization}

LBNs were prepared using carboxyfluorescein-labeled DOPE and the rhodamine labeled pDNA. The rMSCs were incubated in 96-well plates at $37^{\circ} \mathrm{C}$. The cells were then transfected with dye-labeled LBNs and incubated for $4 \mathrm{~h}$. The transfection medium was then replaced with fresh expansion medium. MSCs were later fixed with $4 \%$ paraformaldehyde for 20 min. Cell nuclei were stained with DAPI. The LBN particle internalization was visualized with fluorescence microscope. 


\subsection{Gene Transfection}

Dulbecco's Modified Eagles Medium (DMEM) containing 15\% fetal bovine serum (FBS) and $1 \%$ antibiotic was prepared as expansion medium for rMSCs and rat dermal fibroblasts. Cells were cultured in the 24-well plate at a density of $1 \times 10^{5}$ cells/well and incubated for $24 \mathrm{~h}$. Cell culture medium was replaced with $500 \mu \mathrm{L}$ fresh expansion medium and different LBNs were added with a final $1 \mu \mathrm{g} / \mathrm{mL}$ DNA per well. Lipofectamine 2000 was used as a control. The cells were incubated for another $4 \mathrm{~h}$ at $37^{\circ} \mathrm{C}$. The transfection medium was then replaced with a fresh expansion medium. GFP expression was visualized with fluorescent microscopy. For flow cytometry evaluation (Becton-Dickinson Biosciences, Franklin Lakes, NJ, U.S.A.), the transfected cells were detached from culture flasks by trypsin digestion and fixed in $4 \%$ paraformaldehyde for 20 min to prepare single cell suspensions.

\subsection{Immunofluorescence Assay}

A $4 \%$ fresh paraformaldehyde was used to fix rMSCs for $40 \mathrm{~min}$ at room temperature. Then PBS was used to wash the cells, followed by incubation of the cells for $1 \mathrm{~h}$ in blocking buffer (5\% goat serum, 1\% BSA, 0.1\% Triton X-100 in PBS). After the blocking buffer was removed, the cells were incubated overnight at $4{ }^{\circ} \mathrm{C}$ in the presence of primary antibody (VEGF, rabbit, abcam) diluted in PBS (5\% goat serum). Then PBS was used to wash the cells. The cells were then incubated with the dye-labeled antibody, goat antirabbit IgGTRITC (1:250, Santa Cruz Biotechnology) in PBS (5\% goat serum), without light at room temperature for $2 \mathrm{~h}$. After the cells were washed, they were stained with DAPI $(0.5 \mu \mathrm{g} / \mathrm{mL}$, Invitrogen). A fluorescence microscope was then used to image the cells.

\section{13. Statistical Analysis}

Statistical analysis was performed using two-tailed unpaired Student's $t$ test. Data are presented as mean \pm standard deviation.

\section{RESULTS AND DISCUSSION}

LBNs were fabricated using a packaging technique as described in Figure 1. Briefly, the negatively charged DNA, i.e., the SB transposon system, was condensed via electrostatic interactions with positively charged protamine. The DNA to protamine ratio was tuned to form a slightly negatively charged polyplex. The rMSC-targeting peptides were conjugated to 1,2-distearoyl-sn-glycero-3-phosphoethanolamine- $\mathrm{N}$-[maleimide-(polyethylene glycol)-2000] (DSPE-PEG-MAL) lipids (Figure 1A). Specifically, the thiol groups on the cysteine residues of 3VT-peptide or VT-peptide were reduced for facilitating the conjugation of the targeting peptide to DSPE-PEG-MAL lipid. The cysteine reduction efficiency was $>95 \%$, as determined by DTNB assay. ${ }^{40}$ The conjugated anionic peptide-lipid complexes were later anchored onto the LBNs. The resultant peptide-lipid conjugates were coinserted into DOTAP/DOPE/Chol liposomes, which were prepared by a thin film hydration method, ${ }^{41,42}$ using a post-insertion technique. ${ }^{43,44}$ Finally, the positively charged liposome complex was coupled with negatively charged protamine/DNA polyplex to give rise to LBNs (Figure 1B). 
The dynamic light scattering of the as-prepared liposomes (with $0 \%$ peptide-lipid insertion) showed a size of $110.37 \mathrm{~nm}$ with a zeta potential of $77.17 \mathrm{mV}$ (Figure 2). The size of the targeted LBNs (with 10\% 3VT-peptide-lipid insertion) increased to $190.24 \mathrm{~nm}$ while their zeta potential decreased to $62.22 \mathrm{mV}$ (Figure 2). As the molar percentage of peptide-lipid in LBNs further increased, the size of particles increased and the zeta potential continued to decrease, indicating the successful insertion of peptide-lipid into the liposomes to form LBNs. The particle size and zeta potential of LBNs with different percentages of VTpeptide-lipid showed a similar pattern (Supporting Information, Figure S1).

The DNA loading capability of protamine was determined by gel retardation assay (Supporting Information, Figure S2). DNA was completely encapsulated when the mass ratio of protamine/DNA reached 4:1. Thus, 2:1 ratio was used to render the negative charge of protamine/DNA. DNA loading capacity of liposomes was also evaluated (Supporting Information, Figure S3). The result indicated DNA was completely encapsulated and the resultant liposome/DNA complex was slightly positively charged when liposome/DNA mass ratio reached 3:1. Thus, this ratio was used in the subsequent experiments. The positively charged liposome/DNA complex might interact with negatively charged cell surface to favor subsequent endocytosis pathway. ${ }^{45-47}$

The cytotoxicity of as-prepared LBNs was evaluated by MTT assay (Supporting Information, Figure S4) with Lipofectamine 2000 as a control. At a concentration of 20 $\mu \mathrm{g} / \mathrm{mL}$, rMSCs viability was obviously reduced below $60 \%$ with Lipofectamine treatment, whereas all rMSCs remained above $80 \%$ viability when treated with different LBNs. The results clearly demonstrated that our LBNs are more biocompatible than Lipofectamine. Actually, LBNs with a concentration of only $3 \mu \mathrm{g} / \mathrm{mL}$ were used for gene transfection.

After the successful synthesis of LBNs with low toxicity and high DNA-loading capability, we performed cell internalization of LBNs in rMSCs (Figure 3). To verify the capability of internalization of LBNs in rMSCs due to the presence of rMSC-targeting 3VT-peptide on the surface, green dye-labeled lipids and red dye-labeled DNA were used to form LBNs, followed by interaction with rMSCs. Green fluorescence from carboxyfluorescein-labeled liposomes and red fluorescence from rhodamine-labeled DNA demonstrated that the LBNs were successfully internalized in rMSCs. LBNs with the targeting 3VT-peptide showed higher fluorescence than those without a targeting peptide (Figure 3). This result indicated that targeting peptides facilitated the cellular internalization of LBNs. Thus, the rMSCtargeting peptides on the surface of LBNs facilitated the internalization of LBNs in rMSCs, which could lead to enhanced gene delivery to rMSCs. This result is consistent with our earlier discovery in the study where we used phage protein displaying a targeting peptide for enhanced gene delivery to rMSCs. ${ }^{40} \mathrm{We}$ thus hypothesize that the targeting peptide, particularly when in the reiterated form, could promote the receptor-mediated endocytosis of the LBNs, although so far, we have not known what receptors this targeting peptide is recognizing.

The LBNs containing EGFP-VEGF-encoding SB transposon system and rMSCs-targeting peptide were investigated for targeted gene transfection to rMSCs in vitro. The quantitative EGFP expression was determined by flow cytometry. To determine the optimal gene 
transfection efficiency of LBNs, various molar percentages of VT-peptide-lipid (VT-lipid) or 3VT-peptide-lipid (3VT-lipid) in LBNs (without NLS peptide) were evaluated (Figure 4A). As the percentage of peptide-lipid increased, the gene transfection efficiencies also increased. At 5\% of VT-lipid or 3VT-lipid, the transfection efficiencies plateaued at around $26 \%$ or $33 \%$. So 5\% VT-lipid or 3VT-lipid was used for the subsequent experiments. The plateau of transfection efficiency might result from the more negative zeta potential of LBNs with an increased percentage of peptide-lipid in LBNs, since more negative zeta potential of the nanoparticles will not favor cell internalization. The effect of varying NLS peptide/DNA mass ratio on the transfection efficiency was also monitored with liposomes modified by $5 \%$ 3VT-lipid. A 2:1 peptide/DNA ratio resulted in the highest gene expression (Figure 4B). Therefore, this ratio was used for the following experiments.

Transfection efficiencies of various LBNs were evaluated (Figure 5A). The corresponding EGFP gene expression was visualized with fluorescence microscopy (Figure 5B). We found that LBNs without the surface display of the peptides (13.64\%) showed similar transfection efficiency compared with commercially available Lipofectamin 2000 (14.65\%). However, when displaying VT-peptide, LBNs exhibited an enhanced transfection efficiency (25.64\%). Moreover, LBNs with 3VT-peptide (32.57\%) demonstrated an even higher transfection efficiency in comparison to those with VT-peptide. Importantly, when LBNs contained both 3VT-peptide and NLS peptide, the highest level of transfection (52.52\%) was reached. Our results clearly indicated that both VT-peptide and 3VT-peptide facilitated LBNs to efficiently deliver plasmid gene into MSCs. The 3-fold reiterated 3VT-peptide demonstrated significantly enhanced transfection efficiency compared with VT-peptide. The targeting peptide and NLS peptide exerted a synergetic effect to promote gene transfection.

To evaluate the specificity of LBNs, rat dermal fibroblasts were used as a control cell for gene transfection (Figure S5). Flow cytometry results showed low transfection efficiency and no significant difference between LBNs with (3.34\%) and without targeting peptide (3.97\%). The results indicated that LBNs with rMSCs-targeting peptide had no specificity for rat dermal fibroblasts.

A time course assessment of gene expression was carried out to evaluate the expression of EGFP-encoding SB transposon system for up to $168 \mathrm{~h}$ (Figure 6). The expression of EGFP in both EGFP-encoding SB transposon system and traditional (control) EGFP-encoding plasmid (pEGFP-N1) reached maximum at $72 \mathrm{~h}$. However, the transfection efficiency of control EGFP plasmid later decreased substantially at $168 \mathrm{~h}$. In comparison, the SB system maintained a high transfection level even at $168 \mathrm{~h}$. This result demonstrated the stable and durable gene transfection effect of SB system due to the efficient integration of target gene into rMSCs host genome by the transposase in the SB system.

It was shown that VEGF gene delivery to MSCs improved therapeutic function of MSCs. ${ }^{43,48-50}$ The strategy for constructing EGFP-VEGF-encoding SB transposon system was shown in Scheme S1. After LBNs carrying the SB transposon system were used to transfect rMSCs, immunofluorescence assay was performed to visualize the VEGF gene expression (Figure 7) after $144 \mathrm{~h}$ of expression. When rMSCs were transfected with EGFPVEGF-encoding SB transposon system, high transfection efficiency and coexpression of 
EGFP and VEGF were observed. In comparison, only EGFP gene expression, but no VEGF expression, was detected when control EGFP-encoding SB transposon system was used. This result demonstrated the feasibility of using the LBN system for efficient VEGF gene delivery to rMSCs.

\section{CONCLUSION}

In summary, we developed a LBN system by incorporating the targeting peptides, NLS peptides and a VEGF-encoding SB transposon system. The resultant targeting LBN demonstrated a high gene transfection efficiency and sustained gene expression profile, due to improved MSCs-internalization, nuclear translocation and gene integration. The 3-fold reiterated targeting peptide showed much higher capability in improving the transfection efficiency in stem cells than the nonreiterated targeting peptide. Targeting peptide and NLS peptide as well as the use of SB transposon system exerted a synergetic effect to promote efficient and lasting transfection gene expression. The LBNs are shown to efficiently deliver VEGF-encoding SB transposon system into stem cells to enable the cells to efficiently produce VEGF. This targeting LBN complex may have promising applications in clinical stem cell-based gene therapy.

\section{Supplementary Material}

Refer to Web version on PubMed Central for supplementary material.

\section{Acknowledgments}

We would like to thank the financial support from National Institutes of Health (CA200504 and CA195607), National Science Foundation (CMMI-1234957 and CBET-1512664), Department of Defense office of the Congressionally Directed Medical Research Programs (W81XWH-15-1-0180), Oklahoma Center for Adult Stem Cell Research (434003), and Oklahoma Center for the Advancement of Science and Technology (HR14-160). M.Y. acknowledges the support of Zhejiang Provincial Natural Science Foundation of China (LZ12C17001) and Projects of Zhejiang Provincial Science and Technology Plans (2012C12910), National Natural Science Foundation of China (21172194), Silkworm Industry Science and Technology Innovation Team (2011R50028), and National High Technology Research and Development Program 863 (2013AA102507). We also thank Dr. Kun Ma for the kind help during the experiments.

\section{REFERENCES}

1. Jackson KA, Majka SM, Wang H, Pocius J, Hartley CJ, Majesky MW, Entman ML, Michael LH, Hirschi KK, Goodell MA. J. Clin. Invest. 2001; 107:1395. [PubMed: 11390421]

2. van der Bogt KE, Sheikh AY, Schrepfer S, Hoyt G, Cao F, Ransohoff KJ, Swijnenburg R-J, Pearl J, Lee A, Fischbein M. Circulation. 2008; 118:S121-S129. [PubMed: 18824743]

3. Arinzeh TL, Peter SJ, Archambault MP, Van Den Bos C, Gordon S, Kraus K, Smith A, Kadiyala S. J. Bone Joint Surg. Am. 2003; 85:1927-1935. [PubMed: 14563800]

4. Dupont KM, Sharma K, Stevens HY, Boerckel JD, García AJ, Guldberg RE. Proc. Natl. Acad. Sci. U. S. A. 2010; 107:3305-3310. [PubMed: 20133731]

5. Reya T, Morrison SJ, Clarke MF, Weissman IL. Nature. 2001; 414:105-111. [PubMed: 11689955]

6. Tang C, Ang BT, Pervaiz S. FASEB J. 2007; 21:3777-3785. [PubMed: 17625071]

7. Caplan A. J. Pathol. 2009; 217:318-324. [PubMed: 19023885]

8. Ferrara N, Gerber H-P, LeCouter J. Nat. Med. 2003; 9:669-676. [PubMed: 12778165]

9. Arinzeh TL, Peter SJ, Archambault MP, Van Den Bos C, Gordon S, Kraus K, Smith A, Kadiyala S. J. Bone Joint Surg. Am. 2003; 85:1927-1935. [PubMed: 14563800] 
10. Wang J, Yang M, Zhu Y, Wang L, Tomsia AP, Mao CB. Adv. Mater. 2014; 26:4961-4966. [PubMed: 24711251]

11. Wang Y, Haider HK, Ahmad N, Xu M, Ge R, Ashraf M. J. Mol. Cell. Cardiol. 2006; 40:736-745. [PubMed: 16603183]

12. Matsumoto R, Omura T, Yoshiyama M, Hayashi T, Inamoto S, Koh K-R, Ohta K, Izumi Y, Nakamura Y, Akioka K. Arterioscler., Thromb., Vasc. Biol. 2005; 25:1168-1173. [PubMed: 15831811]

13. Xiong N, Zhang Z, Huang J, Chen C, Jia M, Xiong J, Liu X, Wang F, Cao X, Liang Z. Gene Ther. 2011; 18:394-402. [PubMed: 21107440]

14. Tan G-R, Feng S-S, Leong DT. Biomaterials. 2014; 35:3044-3051. [PubMed: 24439415]

15. Kutty RV, Tay CY, Lim CS, Feng S-S, Leong DT. Nano Res. 2015; 8:1-15.

16. Kutty RV, Chia SL, Setyawati MI, Muthu MS, Feng S-S, Leong DT. Biomaterials. 2015; 63:58-69. [PubMed: 26081868]

17. Subramani K, Hosseinkhani H, Khraisat A, Hosseinkhani M, Pathak Y. Curr. Nanosci. 2009; 5:135-140.

18. Zisa D, Shabbir A, Suzuki G, Lee T. Biochem. Biophys. Res. Commun. 2009; 390:834-838. [PubMed: 19836359]

19. Lin H, Shabbir A, Molnar M, Yang J, Marion S, Canty JM, Lee T. J. Cell. Physiol. 2008; 216:458_ 468. [PubMed: 18288639]

20. Duan C, Liu J, Yuan Z, Meng G, Yang X, Jia S, Zhang J, Chen S. Arch. Med. Sci. 2014; 1:174. [PubMed: 24701231]

21. Hosseinkhani H, He W-J, Chiang C-H, Hong P-D, Yu D-S, Domb AJ, Ou K-L. J. Nanopart. Res. $2013 ; 15: 1-15$.

22. Hosseinkhani H, Hong P-D, Yu D-S, Chen Y-R, Ickowicz D, Farber I-Y, Domb AJ. Int. J. Nanomed. 2012; 7:3035.

23. Yang F, Cho S-W, Son SM, Bogatyrev SR, Singh D, Green JJ, Mei Y, Park S, Bhang SH, Kim B-S. Proc. Natl. Acad. Sci. U. S. A. 2010; 107:3317-3322. [PubMed: 19805054]

24. Park JW, Ku SH, Moon HH, Lee M, Choi D, Yang J, Huh YM, Jeong JH, Park TG, Mok H. Macromol. Biosci. 2014; 14:380-389. [PubMed: 24634264]

25. Liu B, Li X, Liang G, Liu X. Mol. Med. Rep. 2011; 4:1121-1126. [PubMed: 21850376]

26. Yang J, Zhou W, Zheng W, Ma Y, Lin L, Tang T, Liu J, Yu J, Zhou X, Hu J. Cardiology. 2007; 107:17-29. [PubMed: 16741354]

27. Kim H, Nam K, Nam J-P, Kim HS, Kim YM, Joo WS, Kim SW. J. Controlled Release. 2015; 220:222-228.

28. Zhu K, Lai H, Guo C, Xu D, Wang C. Exp. Biol. Med. (London, U. K.). 2012; 237:678-687.

29. Wang W, Li W, Ou L, Flick E, Mark P, Nesselmann C, Lux CA, Gatzen HH, Kaminski A, Liebold A. J. Cell. Mol. Med. 2011; 15:1989-1998. [PubMed: 20629995]

30. Ma K, Wang DD, Lin Y, Wang J, Petrenko V, Mao CB. Adv. Funct. Mater. 2013; 23:1172-1181. [PubMed: 23885226]

31. Tkachenko AG, Xie H, Liu Y, Coleman D, Ryan J, Glomm WR, Shipton MK, Franzen S, Feldheim DL. Bioconjugate Chem. 2004; 15:482-490.

32. Zanta MA, Belguise-Valladier P, Behr J-P. Proc. Natl. Acad. Sci. U. S. A. 1999; 96:91-96. [PubMed: 9874777]

33. Mátés L, Chuah MK, Belay E, Jerchow B, Manoj N, Acosta-Sanchez A, Grzela DP, Schmitt A, Becker K, Matrai J. Nat. Genet. 2009; 41:753-761. [PubMed: 19412179]

34. Dupuy AJ, Akagi K, Largaespada DA, Copeland NG, Jenkins NA. Nature. 2005; 436:221-226. [PubMed: 16015321]

35. Hoare M, Greiser U, Schu S, Mashayekhi K, Aydogan E, Murphy M, Barry F, Ritter T, O’Brien T. J. Gene Med. 2010; 12:207-218. [PubMed: 20082426]

36. Sorgi F, Bhattacharya S, Huang L. Gene Ther. 1997; 4:961-968. [PubMed: 9349433]

37. Li S, Rizzo M, Bhattacharya S, Huang L. Gene Ther. 1998; 5:930. [PubMed: 9813664] 
38. Zhu H, Cao B, Zhen Z, Laxmi AA, Li D, Liu S, Mao CB. Biomaterials. 2011; 32:4744-4752. [PubMed: 21507480]

39. Wang J, Wang L, Li X, Mao CB. Sci. Rep. 2013; 3:1242. [PubMed: 23393624]

40. Gandra N, Wang DD, Zhu Y, Mao CB. Angew. Chem. 2013; 125:11488-11491.

41. Chono S, Li S-D, Conwell CC, Huang LJ. Controlled Release. 2008; 131:64-69.

42. Masuda T, Akita H, Niikura K, Nishio T, Ukawa M, Enoto K, Danev R, Nagayama K, Ijiro K, Harashima H. Biomaterials. 2009; 30:4806-4814. [PubMed: 19520424]

43. Iyer AK, Su Y, Feng J, Lan X, Zhu X, Liu Y, Gao D, Seo Y, VanBrocklin HF, Courtney Broaddus V. Biomaterials. 2011; 32:2605-2613. [PubMed: 21255833]

44. Iden DL, Allen TM. Biochim. Biophys. Acta, Biomembr. 2001; 1513:207-216.

45. Aoyama T, Hosseinkhani H, Yamamoto S, Ogawa O, Tabata Y. J. Controlled Release. 2002; 80:345-356.

46. Hosseinkhani H, Tabata Y. J. Controlled Release. 2005; 108:540-556.

47. Tay CY, Setyawati MI, Xie J, Parak WJ, Leong DT. Adv. Funct. Mater. 2014; 24:5936-5955.

48. Deuse T, Peter C, Fedak PW, Doyle T, Reichenspurner H, Zimmermann WH, Eschenhagen T, Stein W, Wu JC, Robbins RC. Circulation. 2009; 120:S247-S254. [PubMed: 19752375]

49. Pons J, Huang Y, Arakawa-Hoyt J, Washko D, Takagawa J, Ye J, Grossman W, Su H. Biochem. Biophys. Res. Commun. 2008; 376:419-422. [PubMed: 18789891]

50. Moon H-H, Joo MK, Mok H, Lee M, Hwang K-C, Kim SW, Jeong JH, Choi D, Kim SH. Biomaterials. 2014; 35:1744-1754. [PubMed: 24280192] 


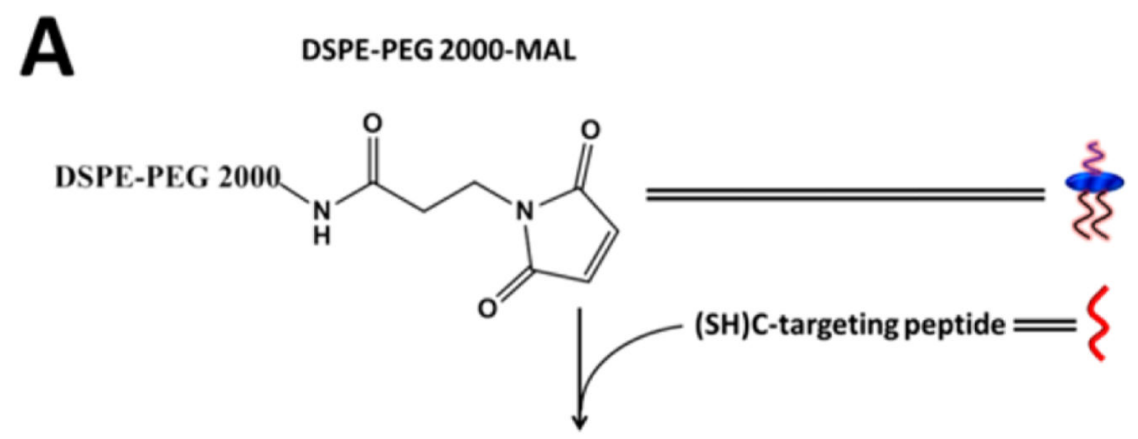

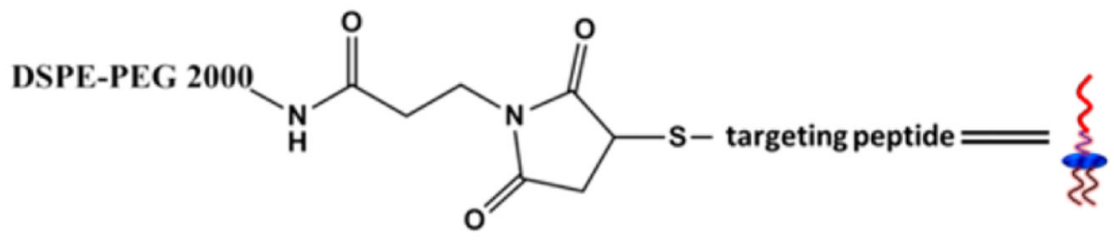

DSPE-PEG 2000-peptide

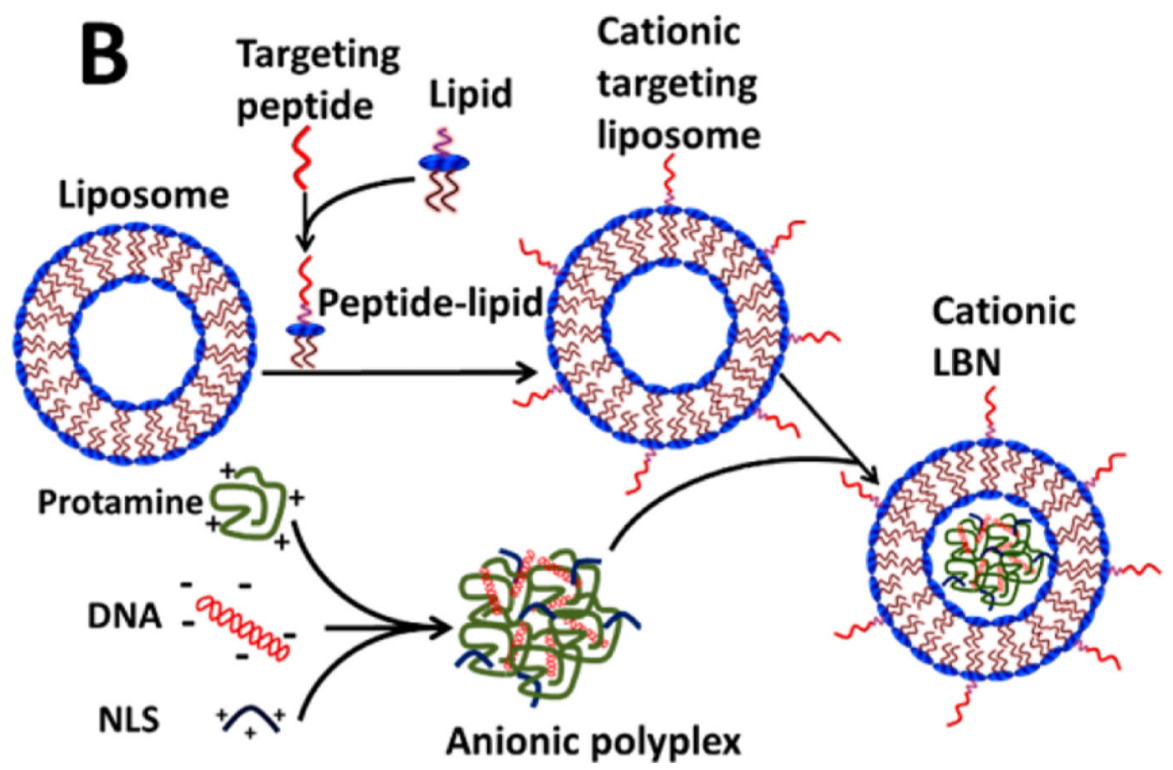

Figure 1.

Schematic showing the construction of stem cell targeting lipid-based nanoparticles (LBNs). (A) Thiol group of cysteine at the carboxyl-terminus of targeting peptide was conjugated to maleimide (MAL) group of DSPE lipid to form peptide-lipid complexes. (B) Peptide-lipid complexes were coassembled with other lipids (DOTAP, DOPE) and cholesterol to form cationic targeting liposomes. Cationic protamine and NLS were collectively used to condense DNA to form anionic protamine/DNA/NLS polyplexs. Finally, the anionic 
polyplexs were entrapped into cationic targeting liposomes to generate cationic targeting LBNs. 
$\multimap$ Size $(\mathrm{nm}) \quad$ Zeta-potential $(\mathrm{mV})$

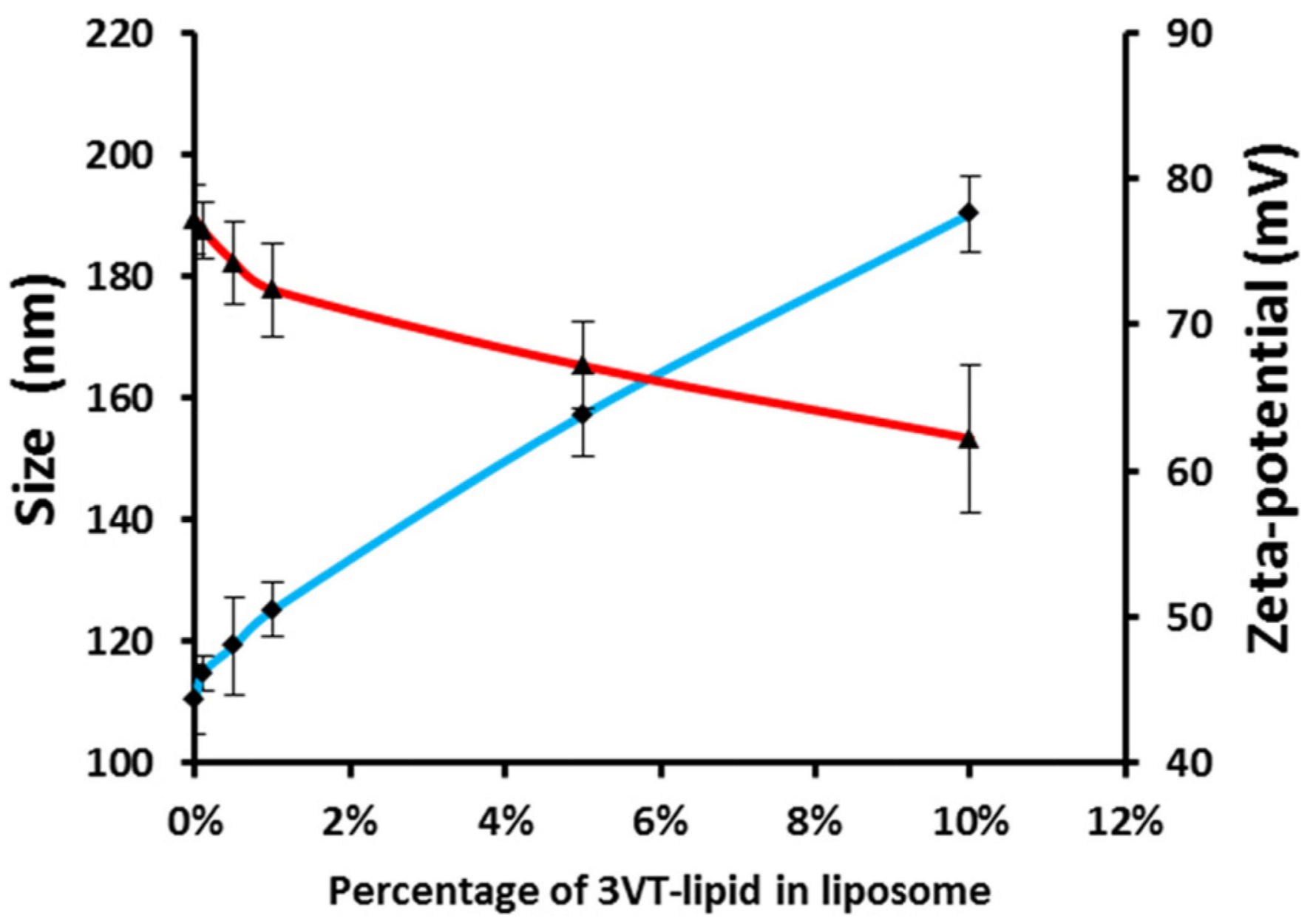

Figure 2.

Comparison of nanoparticle size and zeta potential between liposomes with different molar percentages of 3VT-peptide-lipid. Data are shown as mean \pm standard deviation $(n=3)$. 


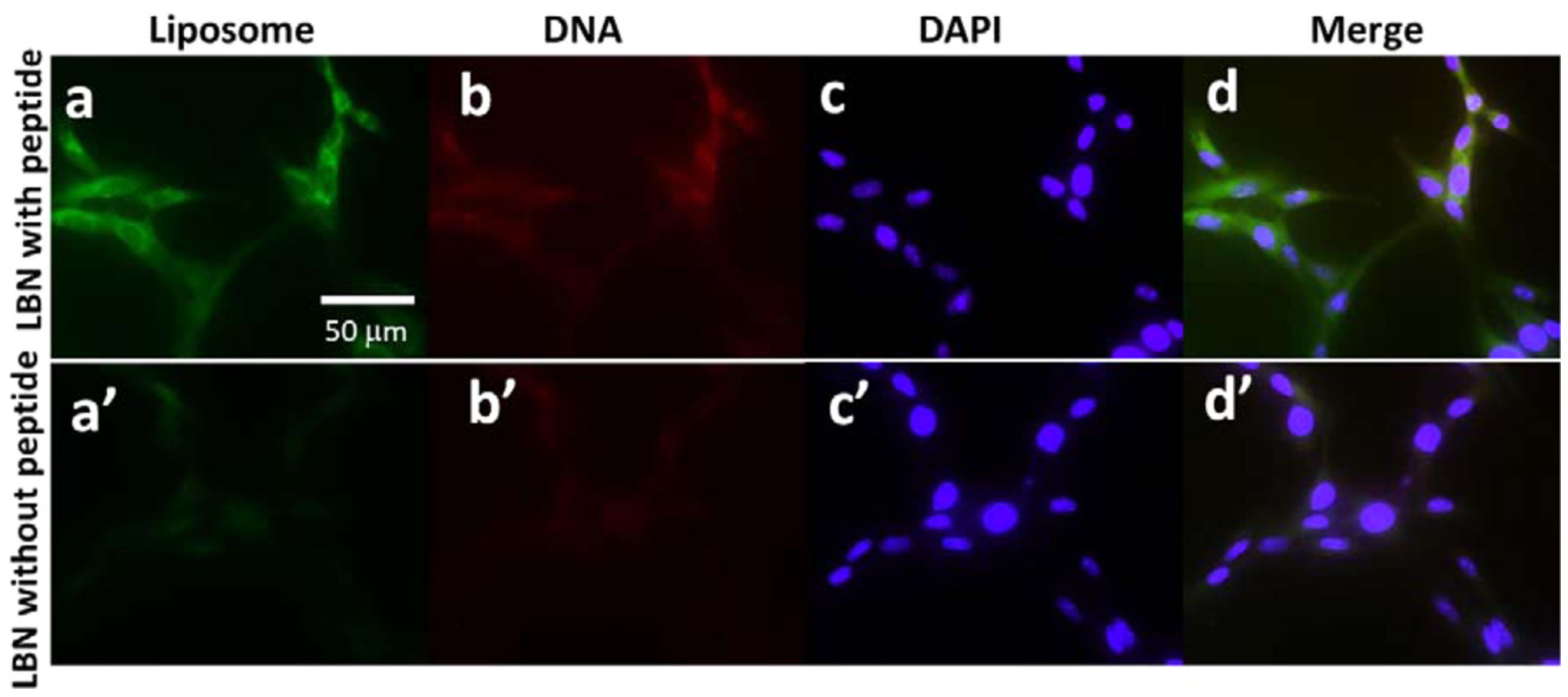

Figure 3.

Fluorescent images of rMSCs after internalization of LBNs with and without 3VT-peptide for $4 \mathrm{~h}$. Lipids, DNA, and cell nuclei were labeled with a green dye (carboxylfluorescein, a and $\mathrm{a}^{\prime}$ ), a red dye (rhodamine, $\mathrm{b}$ and $\mathrm{b}^{\prime}$ ), and DAPI ( $\mathrm{c}$ and $\mathrm{d}^{\prime}$ ), respectively; $\mathrm{d}$ is a merged version of $a, b$, and $c ; d^{\prime}$ is a merged version of $a^{\prime}, b^{\prime}$, and $c^{\prime}$. 

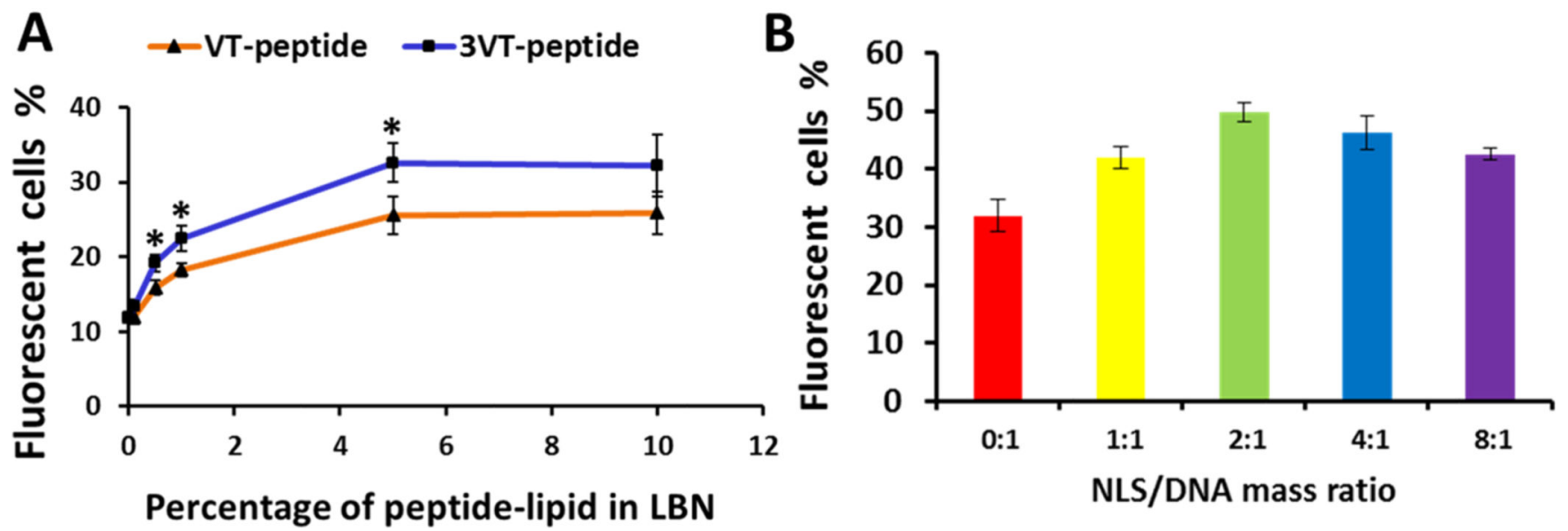

Figure 4.

Effect of rMSC-targeting and NLS peptides on the transfection efficiency in rMSCs by LBNs. (A) The EGFP transfection efficiency of LBNs with various molar percentages of peptide-lipid. Asterisk denotes $p<0.05$ when comparing the transfection efficiency of 3VTpeptide-lipid with VT-peptide-lipid at the same percentage of peptide-lipid. (B) The EGFP gene transfection efficiency of LBNs with various NLS peptide/DNA mass ratios. EGFP gene expression was evaluated after $72 \mathrm{~h}$ transfection. Data are shown as mean \pm standard deviation $(n=3)$. 


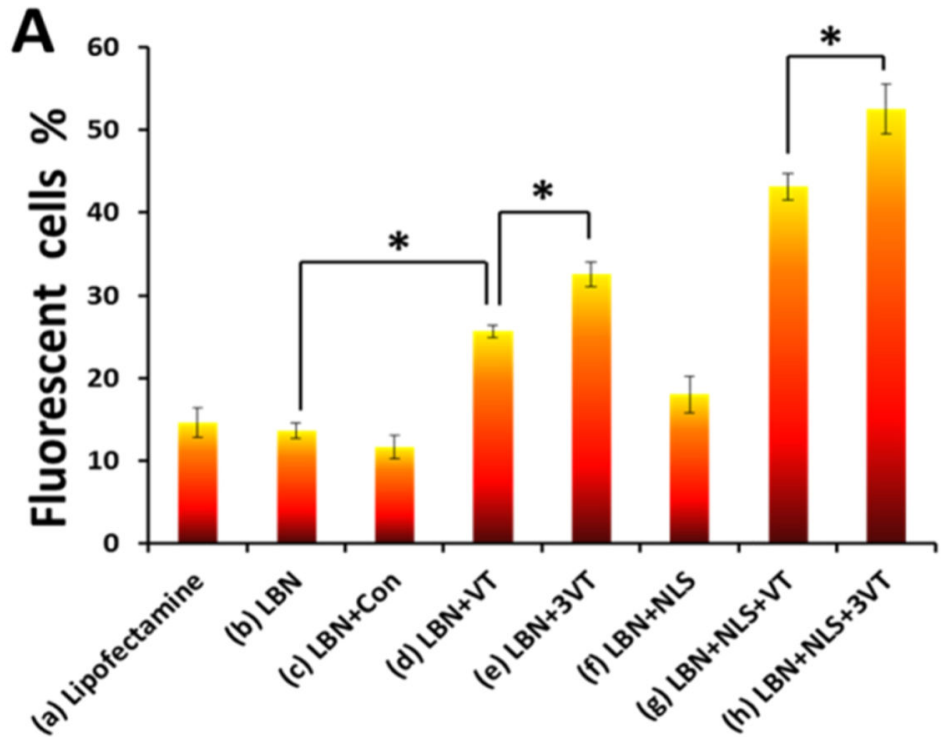

B
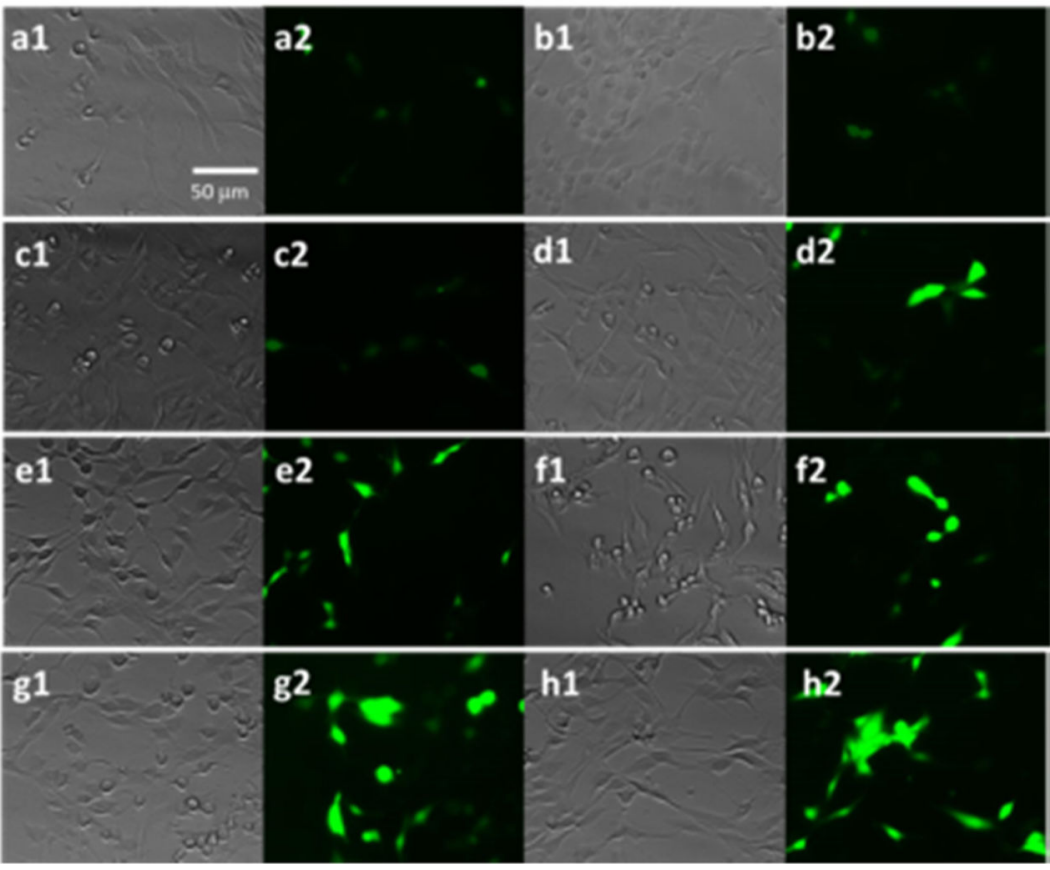

Figure 5.

Comparison of transfection expression of EGFP in rMSCs by different carriers in $72 \mathrm{~h}$. (A) EGFP expression determined by different carriers: (a) Lipofectamine 2000 (control), (b) LBN without any peptide, (c) LBN with control peptide CGVKYMVM, (d) LBN with VTpeptide, (e) LBN with 3VT-peptide, (f) LBN with NLS peptide, (g) LBN with VT-peptide and NLS peptide, and (h) LBN with 3VT-peptide and NLS peptide. Data are shown as mean \pm standard deviation $(n=3)$. Asterisk denotes $p<0.05$. (B) Fluorescence microscopy 
showing EGFP expression in rMSCs by carriers shown in A. Images 1 and 2 in (a)-(h) show the bright-field image and green EGFP expression, respectively. Con denotes control in (A). 


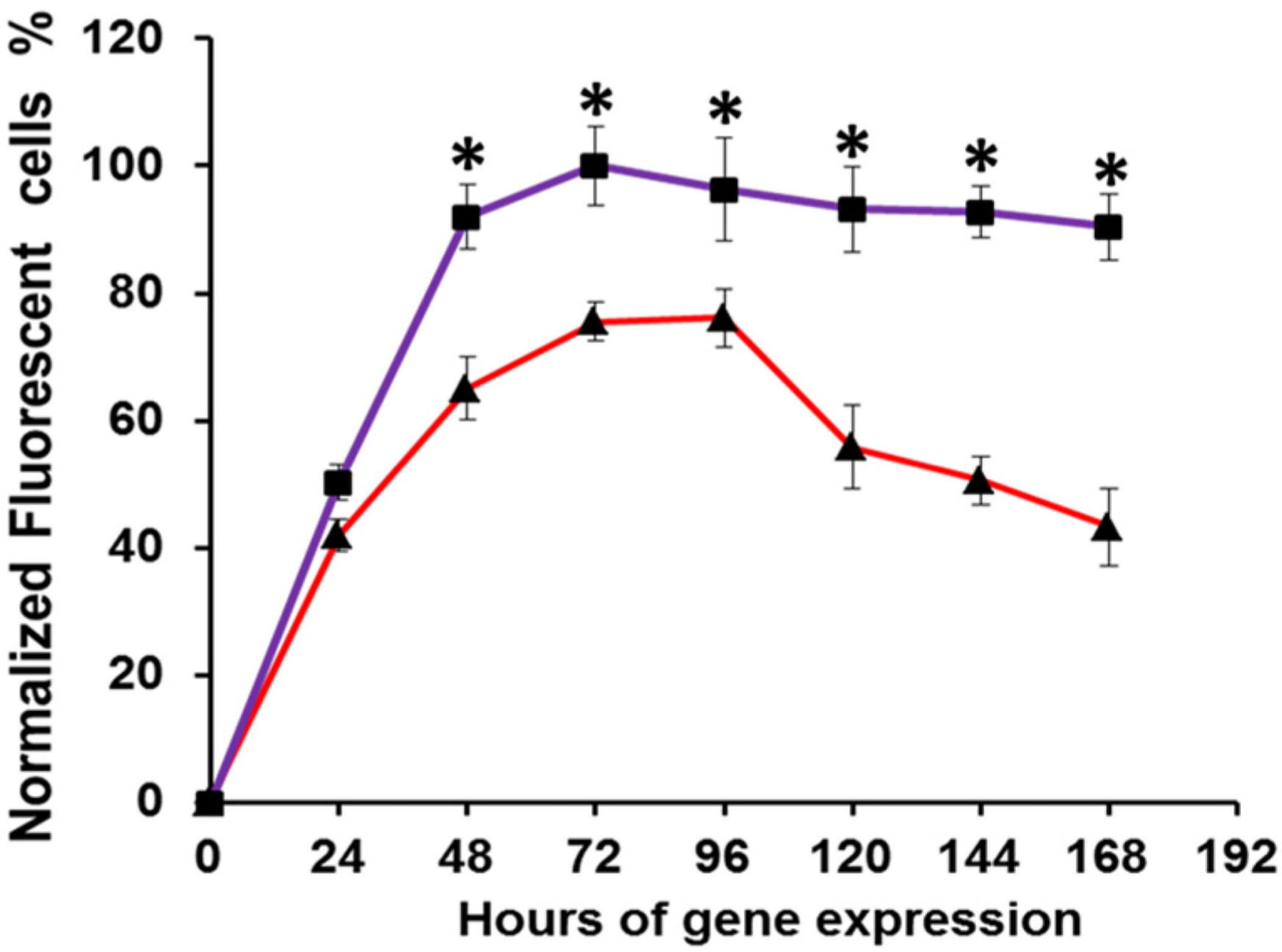

Figure 6.

Time course comparison of gene expression of SB transposon system and control EGFP plasmid showing the long-lasting EGFP gene expression delivered in the form of SB transposon system. EGFP expression level of SB transposon system in rMSCs reached a maximum in $72 \mathrm{~h}$, which was set as $100 \%$. Data are shown as mean \pm standard deviation ( $n$ $=3$ ). Asterisk denotes $p<0.05$ when comparing the gene expression of SB transposon system with control EGFP plasmid. 


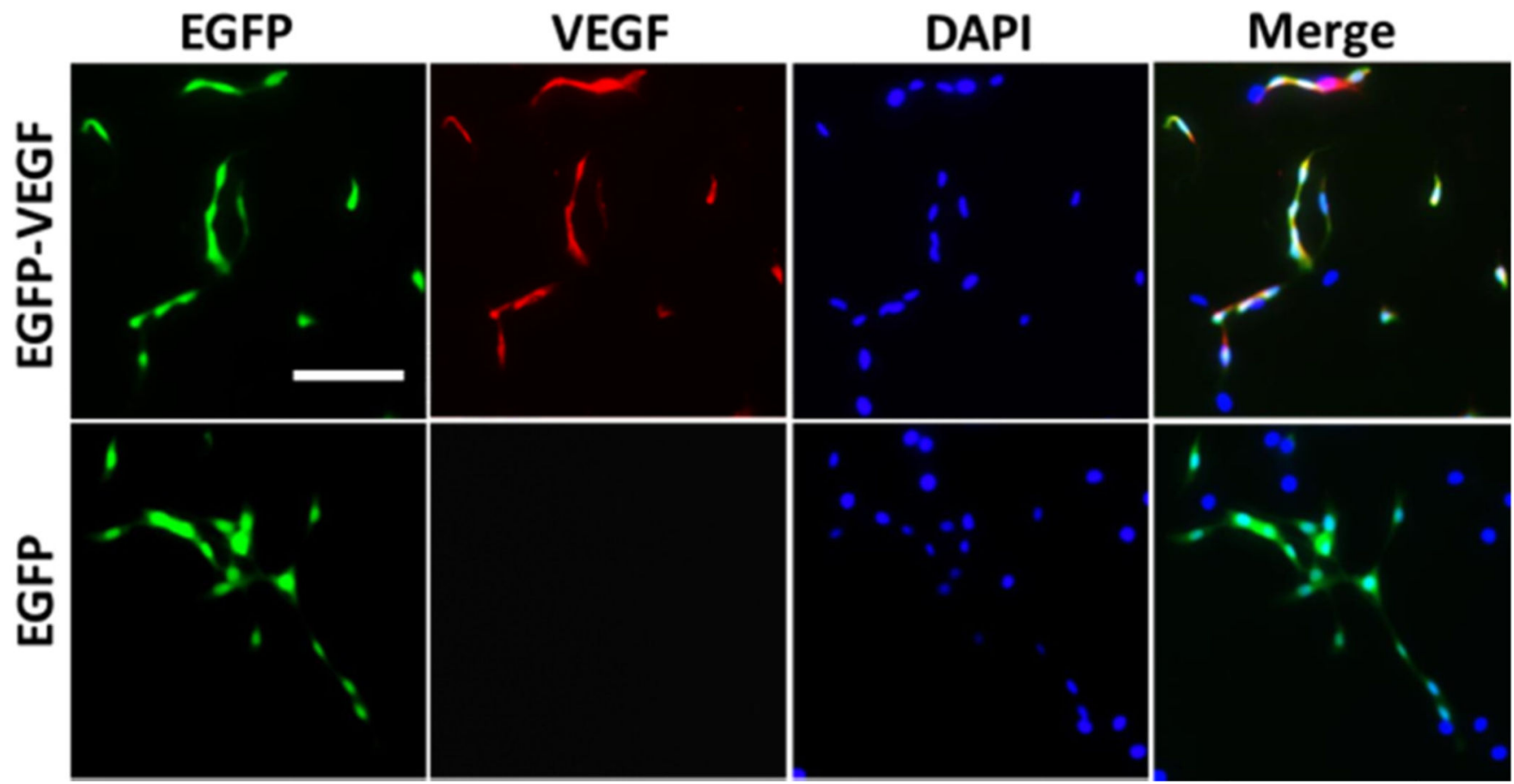

Figure 7.

Comparison of gene expression of EGFP-VEGF or EGFP in SB transposon system by immunofluorescence assay. EGFP gene expression showed green fluorescence. VEGF gene expression was marked with red fluorescent tetramethylrhodamine (TRITC) labeled antibody. Cell nucleus was labeled with blue DAPI. LBNs with 3VT-peptide were used to transfect rMSCs. Scale bar: $50 \mu \mathrm{m}$. 